\title{
Leistungsfähigkeit der Flat-Panel-Technology in 2-D/3-D
}

\author{
Peter Hinnerk Richter, Alexander Grafenberg, Michael Bodky, Christian Schmidgunst, \\ Ina Schwabenland
}

\section{Zusammenfassung}

C-Bögen werden flächendeckend intraoperativ in der Unfallchirurgie und Orthopädie eingesetzt. Neue, minimalinvasive Operationsverfahren verlangen nach einer Optimierung der Bildgebung. In den letzten Jahren kam es zur Etablierung von FlachdetektorC-Bögen, welche entweder stationär oder mobil zur Verfügung stehen. Dies führte zu einer Optimierung der Bildqualität und einer Vergrößerung des Bildvolumens. Gerade für schwierige anatomische Regionen, komplexe Operationsverfahren oder anspruchsvolle minimalinvasive Eingriffe, auch in Kombination mit einem Navigationssystem, sind stationäre C-Bögen in einem Hybridoperationssaal besonders geeignet. Diese neuartigen C-Bögen eignen sich ausgezeichnet für die interdisziplinäre Nutzung. Die Zukunft der intraoperativen Bildgebung liegt in der Flachdetektorbildgebung.

\section{Capability of flat-panel-technology in 2-D/3-D}

C-arms are widely-spread in the operating rooms all-over the world especially in orthopedics. New minimal-invasive operation techniques required an optimization of the imaging devices. Lately flat-panel-c-arms were established in the operating rooms. Not only did this new technology lead to an improvement of image quality but also to a larger field of view. Especially hybrid-operating theatres with a fixed C-arm system are very convenient for difficult anatomical regions, complex fracture patterns and challenging procedures. A combination with a navigation system is possible. Flat-panel-c-arms are suitable for interdisciplinary utilization. The future of intraoperative imaging lies in the flat-panel-technology.

\section{Einleitung}

Weltweit werden C-Bögen flächendeckend in den Operationssälen eingesetzt. Nahezu jede operative medizinische Fachrichtung nutzt heutzutage diese Technologie. Gerade aus dem Arbeitsalltag der Unfallchirurgen und Orthopäden ist die intraoperative Bildgebung nicht mehr wegzudenken. An der Weiterentwicklung und Verbesserung der C-Bögen wird seit vielen Jahren gearbeitet. Besonders für die Etablierung neuer, minimalinvasiver Operationsverfahren war eine Optimierung der intraoperativen Bildgebung unausweichlich. Die Entwicklung von C-Bögen mit Flachdetektor-

OP-JOURNAL 2014; 30: 169-177

(c) Georg Thieme Verlag KG Stuttgart · New York DOI http://dx.doi.org/10.1055/s-0034-1396208

technologie erbrachte hierbei eine signifikante Verbesserung in der intraoperativen Bildgebung. Dies führte nicht nur zu einer Aufwertung der Bildqualität, sondern zu einer zusätzlichen Vergrößerung des Bildvolumens. Zunächst konnte dies nur in stationären C-Bögen integriert werden. Mittlerweile sind mobile C-Bögen mit Flachdetektortechnologie verfügbar. Durch ihre mannigfaltigen Verwendungsmöglichkeiten sind diese C-Bögen für die unterschiedlichsten operativen Verfahren hervorragend geeignet. Neue innovative Operationsverfahren verlangen zusätzlich eine Optimierung der Arbeitsabläufe sowie eine interaktive Vernetzung der unterschiedlichen intraoperativen Geräte. Dadurch kam es in den letzten Jahren zu einer zunehmenden Verbreitung von Hybridoperationssälen. Hierbei wird ein stationäres Bildgebungssystem in einen Operations- saal integriert. Mittels dieser Flachdetektorgeräte können 2-D-Bilder akquiriert werden, welche im Anschluss zu einem 3-D-Bild zusammengesetzt werden können. Im Gegensatz zu den üblichen 3-DStandard-C-Bögen bietet dieses Verfahren eine CT-ähnliche Qualität. Zudem kann dieser 3-D-Scan deutlich schneller durchgeführt werden. In Kombination mit einem Navigationssystem können so komplexe Operationsverfahren an schwierigen anatomischen Regionen durchgeführt werden. Ziel dieses Artikels ist es, die Leistungsfähigkeit der Flachdetektortechnologie zu demonstrieren.

\section{Teil 1: Mobile C-Bögen mit Flachdetektorentechnologie}

Mobile C-Bögen sind seit Jahrzehnten in der Chirurgie im Einsatz. Die intraoperative Bildgebung unterstützt die Chirurgen bei der Visualisierung von anatomischen Strukturen und Implantaten sowie bei der Qualitätssicherung. Der Trend zur minimalinvasiven Chirurgie ist für die Entwicklung von mobilen CBögen insofern wichtig, als diese bildgebenden Geräte durch die Mobilität, die Kompatibilität mit bestehender OP-Infrastruktur und die Kostenposition eine zentrale Rolle im OP spielen. Dabei wird die Präzision und Qualität der Bilder immer wichtiger. Die Integration der Flachdetektortechnologie in die mobilen CBögen trägt diesem Trend Rechnung. Diese Detektoren wurden zu Beginn des Jahrtausends anstelle von Bildwandlern zunächst in die stationären High-End-CBögen und dann in die mobilen C-Bögen integriert. Zusätzlich zur Bildqualität geht der Trend bei den mobilen C-Bögen zur einfacheren Handhabung und Motorisierung (Abb. 1). 

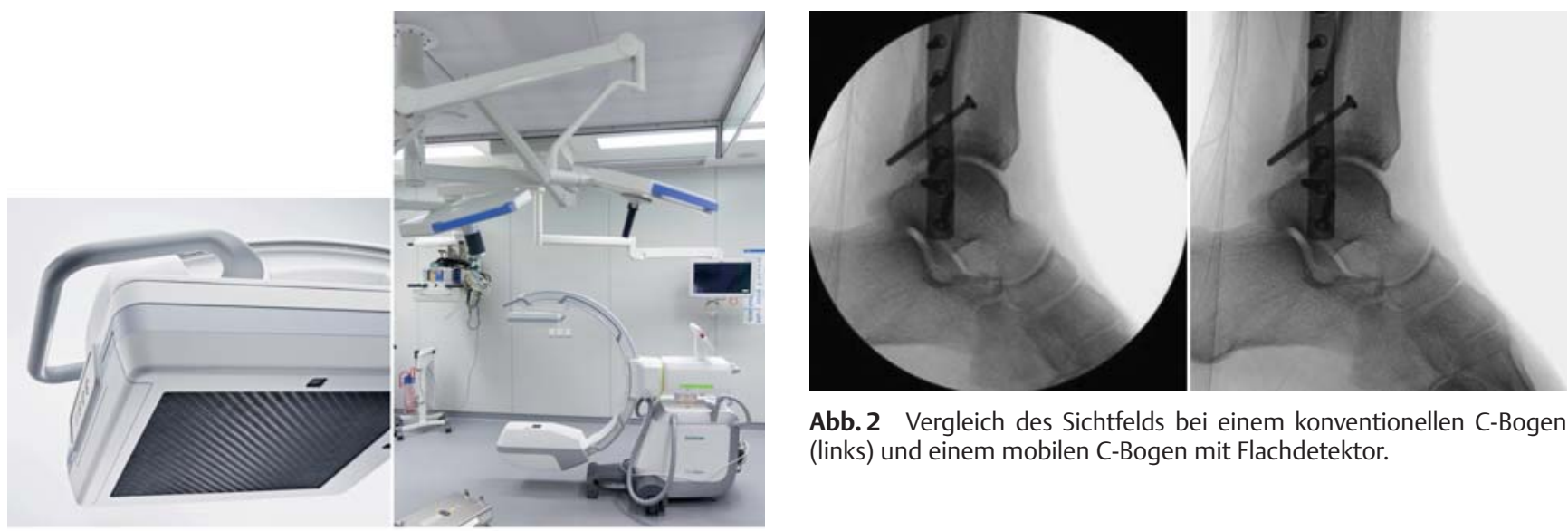

Abb. 2 Vergleich des Sichtfelds bei einem konventionellen C-Bogen (links) und einem mobilen C-Bogen mit Flachdetektor.
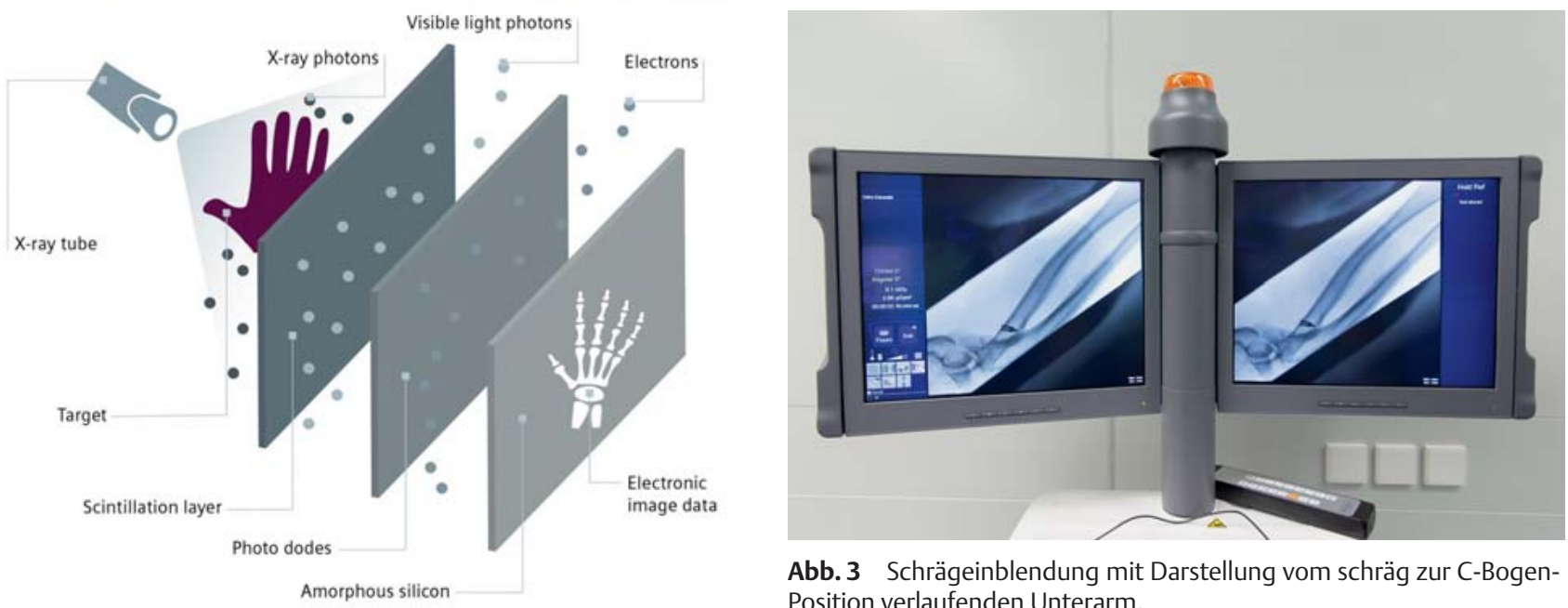

Abb. 3 Schrägeinblendung mit Darstellung vom schräg zur C-BogenPosition verlaufenden Unterarm.

Abb. 1 Schematische Darstellung eines Flachdetektors mit indirekter Umwandlung von Röntgenstrahlung auf Basis von Cäsiumjodid (Csl) und einer aktiven Matrix aus amorphem Silizium (a-Si).

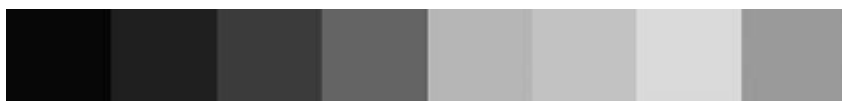

Abb. 4 Schematische Darstellung der Grauskala von Bildverstärkern.

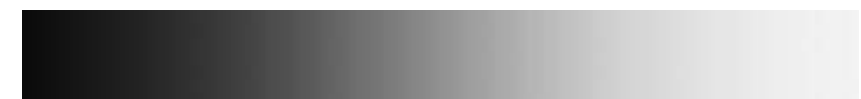

Abb. 5 Schematische Darstellung der Grauskala von Flachdetektoren, $4 \times$ höher.

Klinische Vorteile der mobilen C-Bögen mit Flachdetektor

\section{Sichtfeld}

Wenn die Flachdetektortechnologie (FD-Technologie) mit einem intelligenten Blendensystem ausgestattet ist, liefert sie rechteckige Bilder eines Bereichs von $30 \times 30 \mathrm{~cm}$ und bietet damit eine um bis zu $25 \%$ größere Abdeckung als konventionelle Bildverstärker.

Dies ermöglicht die Visualisierung von anatomischen Regionen in der unmittelbaren Umgebung des Operationsfelds sowie von größeren Teilen langer Knochen. Bei Schrägeinblendung sind die Darstellung von schräg zur C-Bogen-Position verlaufenden Strukturen und die Dre- hung des Bildes in die anatomische Standardansicht möglich (Abb. 2 und 3).

Grauskala und Differenzierung von Strukturen

Die Grauskala moderner Flachdetektoren ist etwa 4-mal so groß wie jene konventioneller Bildverstärker. Der daraus resultierende höhere Kontrast ermöglicht dem Chirurgen, anatomische Strukturen besser zu unterscheiden (Abb. 4, 5, 6).

\section{Mehr Pixel, höhere Genauigkeit}

Konventionelle C-Bögen mit Bildverstärkern liefern Bilder mit 1024 × 1024 Pixel, C-Bögen mit modernen Flachdetektoren Bilder mit $1535 \times 1535$ Pixel.
Die höhere Pixelzahl kombiniert mit der feineren Graustufendifferenzierung und dem größeren Dynamikbereich sorgt für eine höhere Präzision der intraoperativen Bilder (Abb. 7).

\section{Magnetfelder und Bildverzerrung}

Magnetfelder, selbst das der Erde, beeinflussen die Funktion der Elektroden der Bildverstärkerröhre. Infolgedessen können die Bilder insbesondere in den Randbereichen Verzerrungen aufweisen, zusätzlich können in diesen Bereichen die Bilder unscharf sein. Dieses Problem tritt bei mobilen C-Bögen mit modernen Flachdetektoren nicht mehr auf (Abb. 8).

Die Bilder sind klarer und verzerrungsfrei. 

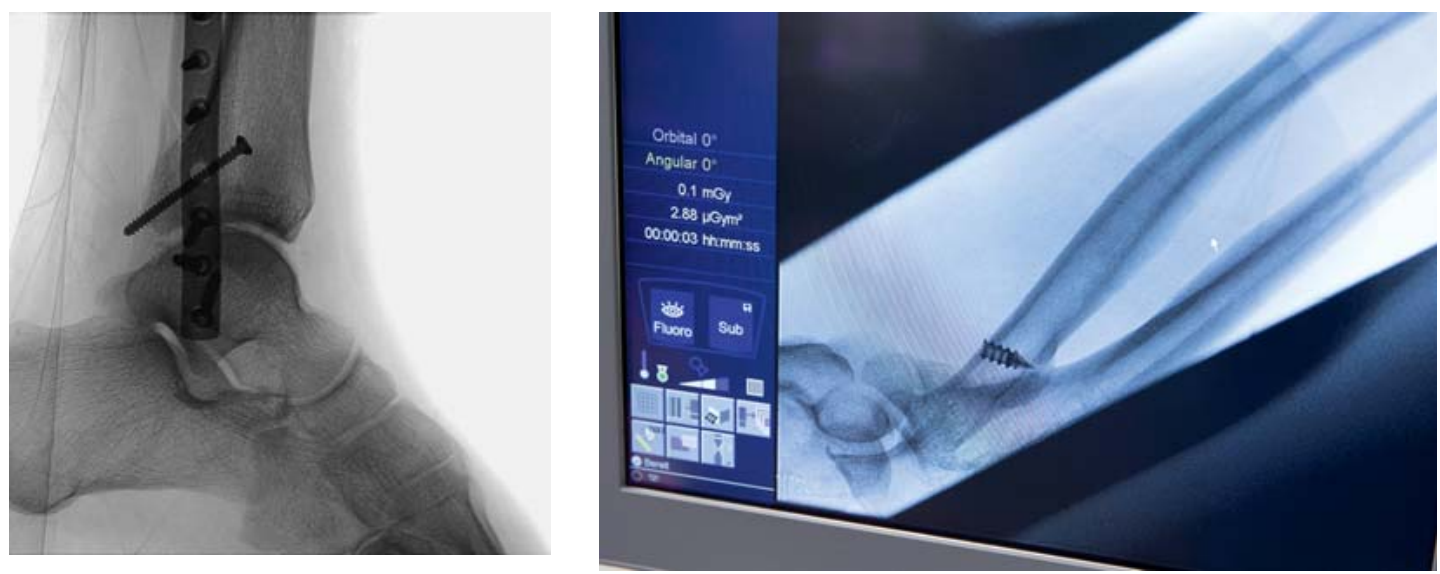

Abb. 7 Bild einer 5-mm-Schraube, aufgenommen mit einem mobilen C-Bogen mit Flachdetektor.

Abb. 6 Eine höhere Grauskala führt zum höheren Kontrast und zu besserer Unterscheidung anatomischer Strukturen von Implantaten.
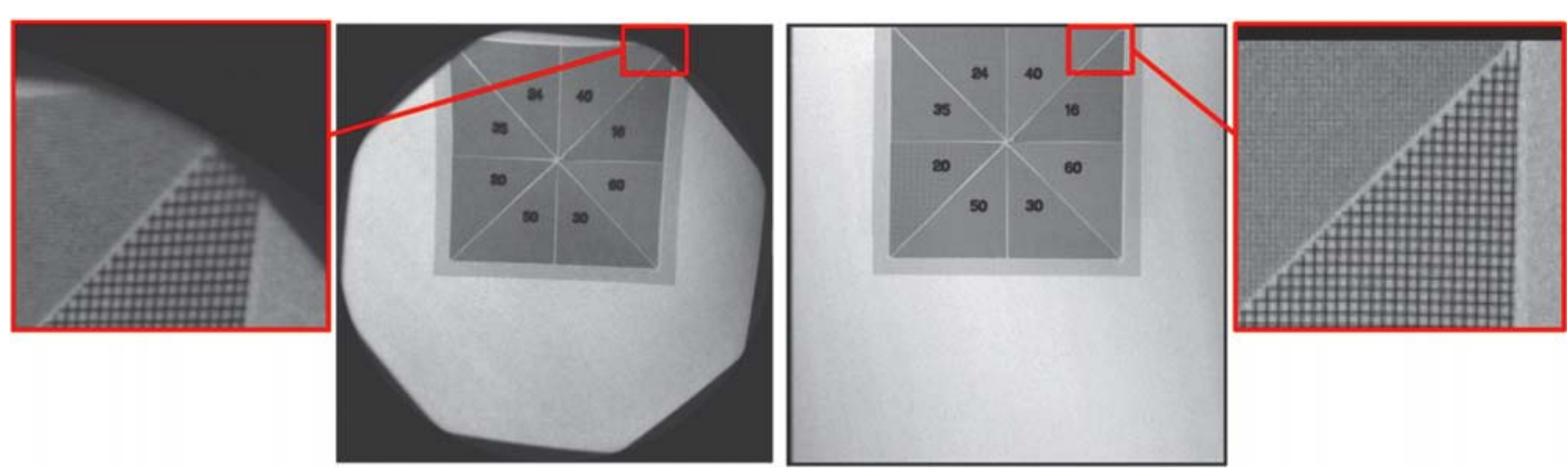

Abb. $\mathbf{8}$ a und $\mathbf{b}$ a Verzerrtes Bild eines Bildverstärkers. b Verzerrungsfreies Bild eines Flachdetektors.

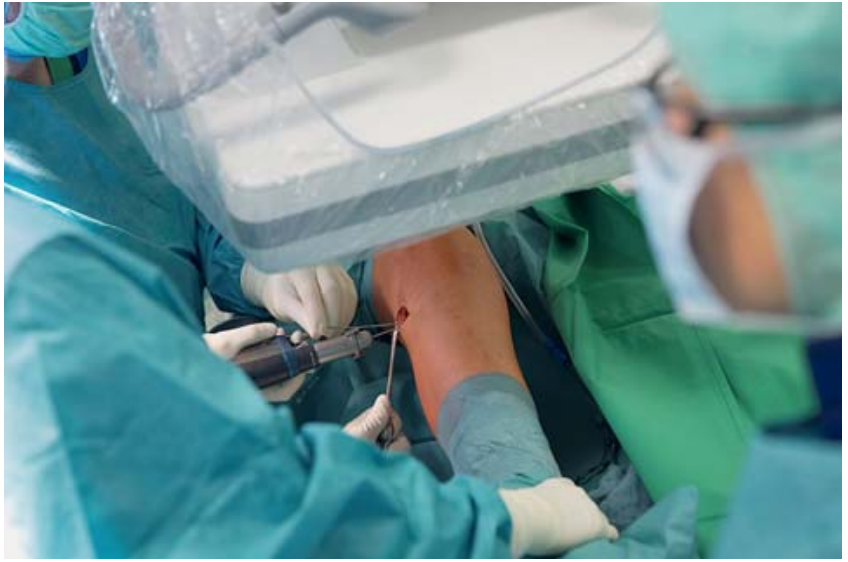

Abb. 9 Durch den Raumgewinn von ca. $10 \mathrm{~cm}$ hat der Chirurg mehr Bewegungsfreiheit bei Eingriffen, z. B. zur Handhabung chirurgischer Instrumente.

Weniger Platzbedarf für den C-Bogen, mehr Platz für den Chirurgen

Der Abstand zwischen dem Fokuspunkt der Röntgenquelle und dem Bildempfänger wird als Fokus-Bildempfänger-Abstand (engl. „Source to Image Distance“, SID) bezeichnet. Bei konventionellen CBögen mit Bildverstärker beträgt dieser (Abb. 9).

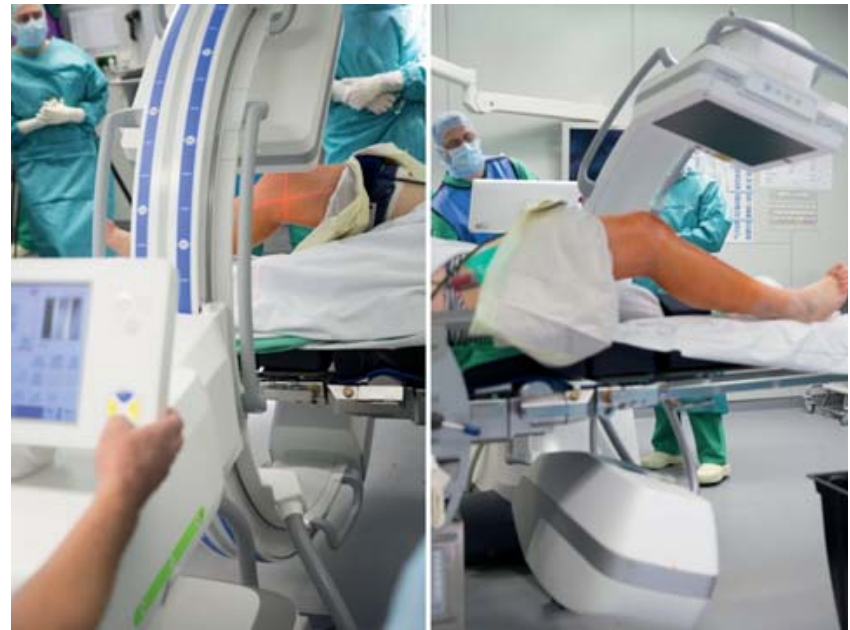

Abb. 10 Präoperative motorische Bewegung des mobilen C-Bogens und Speicherung von wichtigen sich wiederholenden Positionen per Knopfdruck. nologie geht mit Innovationen auch im Bereich der Bedienbarkeit, Motorisierung und dem Workflow einher. So liegt ein weiterer Fortschritt darin, die intraoperative Bildgebung selbst steuern und kontrollieren zu können.

\section{Entscheidende Verbesserungen bei Bedienbarkeit und Workflow}

Die Einführung von neuen C-BogenPlattformen mit der Flachdetektortech-

Abstand ca. $100 \mathrm{~cm}$, bei mobilen C-Bögen mit modernen Flachdetektoren $110 \mathrm{~cm}$ 


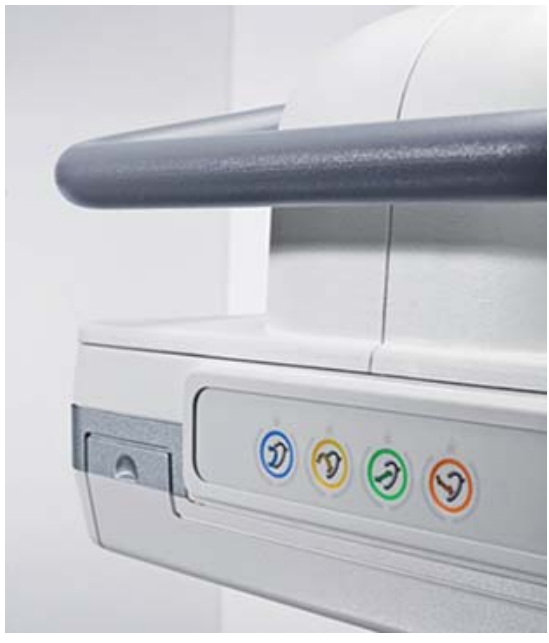

Abb. 11 Bremssteuerung am Flachdetektor ermöglicht dem Chirurgen, den C-Bogen ohne Hilfe Dritter in die gewünschte Position zu manövrieren.

Der Operateur hat nun über einen Touchscreen am OP-Tisch die direkte Kontrolle über die Bilder und die motorische Bewegung des C-Bogens aus dem sterilen Bereich in unmittelbarer Reichweite selbst in der Hand.

Um bspw. Weichteilgewebe zugunsten von Knochenstruktur auszublenden, kann er am Touchscreen die Strahlendosis und Grauwerte ändern. Außerdem ermöglichen Tasten am Flachdetektor zur Bedienung elektromechanischer Bremsen dem Chirurgen, den C-Bogen ohne Hilfe Dritter in die gewünschte Position zu manövrieren, schnell und unkompliziert. Und wenn das Gesamtsystem während des Eingriffs nicht bewegt werden musste, wie bei Hand- und Sprunggelenken oder auch an der Wirbelsäule, kann der C-Bogen auf Knopfdruck in eine gespeicherte Position zurückkehren (Abb. 10-12).

\section{Kollimation}

Moderne mobile C-Bögen mit der Flachdetektortechnologie bieten ein innovatives Kollimationssystem, mit dem Bildausschnitte optimal auf die interessierende Region fokussierbar sind und die Bildqualität zusätzlich gesteigert wird.

Während im konzentrischen Bild älterer C-Bögen nur ein relativ kleiner Knochenabschnitt erschien, können jetzt längere Strecken dargestellt werden, um z.B. Extremitätenachsen zu kontrollieren und Fehlstellungen vorzubeugen. Und mit der asymmetrischen Kollimation lassen sich Strukturen im Randbereich einfan-
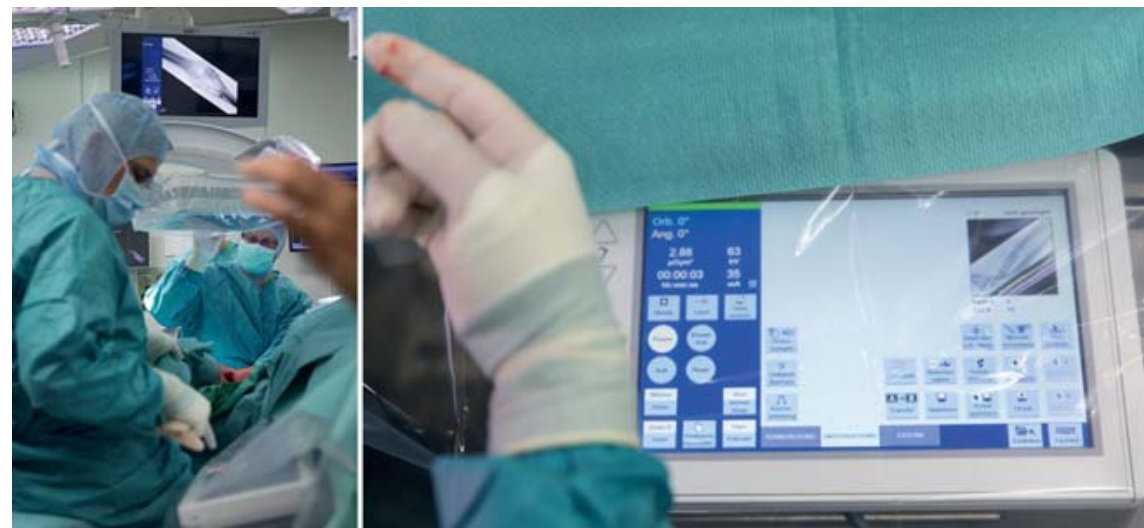

Abb. 12 Der Operateur hat über einen Touchscreen am OP-Tisch die direkte Bildkontrolle aus dem sterilen Bereich selbst in der Hand.

gen, die sonst außerhalb des symmetrischen Bildes liegen würden. Im Idealfall kann das rechteckige Bild aus jeder Richtung, ähnlich einer digitalen Fotobearbeitung, „zugeschnitten“ werden. Damit entfällt oftmals eine Änderung der Patientenposition und die Bildqualität wird durch ausgeblendete Artefakte, wie sie durch das Metall des OP-Tisches entstehen können, verbessert. Zusätzlich kann die Aufnahme rotiert werden, bis sie mit der Knochenposition im Patienten übereinstimmt.

\section{Flachdetektortechnologie und 3-D}

Die $30 \times 30-\mathrm{cm}$-Flachdetektortechnologie bietet darüber hinaus großes Potenzial für den zukünftigen Einsatz in der intraoperativen 3-D-Bildgebung. Der hohe Dynamikbereich der Flachdetektoren verringert die „Truncation-Artefakte" im 3-D-Volumen, was zu einer besseren Darstellung insbesondere an den Objektgrenzen führt. Durch großflächige Flachdetektoren $(30 \times 30 \mathrm{~cm})$ lässt sich ein größeres und optimiertes Volumen rekonstruieren. Eine Verbesserung der 3-D-Navigationsgenauigkeit kann dadurch erzielt werden, dass die durch das Magnetfeld verursachten Bildverzerrungen reduziert werden.

\section{Teil 2: Hybridoperationssaal}

Die Entwicklung minimalinvasiver Techniken und die rasanten Fortschritte in der Chirurgie fordern die Integration fortschrittlicher Bildgebungstechnologien in den Operationssaal. Unter einem Hybridoperationssaal (OP) versteht man den Einbau eines stationären Bildgebungssystems in einen Operationssaal, in dem interventionelle und offene Verfahren kombiniert werden können.
Das Fehlen des Tastvermögens und des natürlichen 3-dimensionalen Sehens bei minimalinvasiven chirurgischen Eingriffen verlangt nach bildgebenden Verfahren und die Visualisierung spielt dabei eine zentrale Rolle. Die Vernetzung unterschiedlicher Bildgebungsverfahren, um Strukturen unterhalb der Oberfläche sichtbar zu machen und chirurgische Instrumente sicher zu führen, ist ein Vorteil dieses neuen Konzepts. Um HybridOPs zu entwerfen, die bestehende und aufkommende Arbeitsabläufe unterstützen, ist ein umfassendes Verständnis der gängigen und zukünftigen Technologien unerlässlich.

Die laufende Weiterentwicklung der interventionellen und chirurgischen Verfahren verlangt nach der Gestaltung eines neuen Arbeitsumfelds, das den interdisziplinären Teams ermöglicht, sich zu integrieren und zu kollaborieren. Vorgestellt wurde dieses neuartige Konzept zum ersten Mal im Jahr 1990, als der erste Hybrid-OP für endovaskuläre Verfahren am Herz-Lungen-Zentrum in Monaco eingerichtet wurde. Herz- und Gefäßchirurgen entwickelten die endovaskulären Behandlungsabläufe zunächst, um degenerierte Herzklappen und Gefäßaneurysmen $\mathrm{zu}$ behandeln. Der allgemeine Trend in der Chirurgie hin $\mathrm{zu}$ minimalinvasiven Verfahren und die engagierten technischen Entwicklungen haben sich jedoch schnell auf nahezu alle chirurgischen Fachgebiete ausgeweitet.

Neue Technologien werden entwickelt, um den speziellen Anforderungen gerecht zu werden. Dazu gehören u.a. eine benutzerfreundliche Bedienoberfläche, Datenverbindungen und Datenanzeige, Bilddatenüberlagerung von mehreren Bildgebungsverfahren und die 3-dimensionale Darstellung der Anatomie in 
Echtzeit, um nur einige zu nennen. Zukünftig werden Operationssäle eine große Bandbreite der zentralen chirurgischen Systeme miteinander vernetzen können. So z.B. Robotertechnik, Röntgengeräte, Navigation, Ultraschall und Endoskopie. Damit zeigt sich, wie wichtig es ist, bei der Gestaltung eines Hybrid-OPs nicht nur gegenwärtige Bedürfnisse und Systeme zu berücksichtigen, sondern auch die sich rasch entwickelnden Therapiemöglichkeiten und zukünftigen Anforderungen mit einzubeziehen. Zur Verfügung steht ein dedizierter, robotergestützter chirurgischer C-Bogen, der, im Vergleich zu herkömmlichen Systemen, für mehr Flexibilität im Operationssaal sorgt. Dieses vom Chirurgen gesteuerte Mehrachssystem bietet volle Flexibilität und Rundumzugang zum Patienten und garantiert somit präzise und schnelle Patientenversorgung, Bildgebung und Parkpositionen eingeschlossen [3] (Abb. 13).

\section{Aufbau eines Hybrid-OPs}

Voraussetzung für einen Hybrid-OP ist die sorgfältige Planung des Raumes, die mit einbezieht, welchen Platz die Bildgebungstechnik und die Eingliederung der unterschiedlichen OP-Komponenten in ein funktionales Gesamtsystem brauchen. Alle Disziplinen sollten schon zu Beginn der Planungsphase in die Projektierung des neuen Operationssaals mit einbezogen werden. Ein multifunktionaler Raum benötigt eine präzisere Gestaltung und ein funktionaleres Arbeitskonzept als ein Standard-OP. Empfohlen wird eine Raumgröße von mindestens $70 \mathrm{~m}^{2}$ oder mehr. Hinzu kommen ein Technik- und ein Schaltraum von $10 \mathrm{~m}^{2}$. Die Planung und Umsetzung eines Hybrid-OPs dauert normalerweise 12-18 Monate, je nachdem, ob ein vorhandener Saal umgebaut oder ein Neubau errichtet werden soll.

Zusätzliche Aspekte sollten bei der Planung des Operationstischs berücksichtigt werden, dazu gehören dessen Position im Raum, die Strahlendurchlässigkeit (Carbon-Tischplatte), die Kompatibilität und die Integration der Bildgebungsgeräte in den Operationstisch, z.B. die von Robotern. Chirurgen, und im Besonderen orthopädische Chirurgen, allgemeine Chirurgen und Neurochirurgen, erwarten i.d. R. einen Tisch mit segmentierter Tischplatte zur flexiblen Patientenlagerung. Die speziell für Operationen entwickelten, frei schwimmenden Angiografietische mit vertikaler und la-

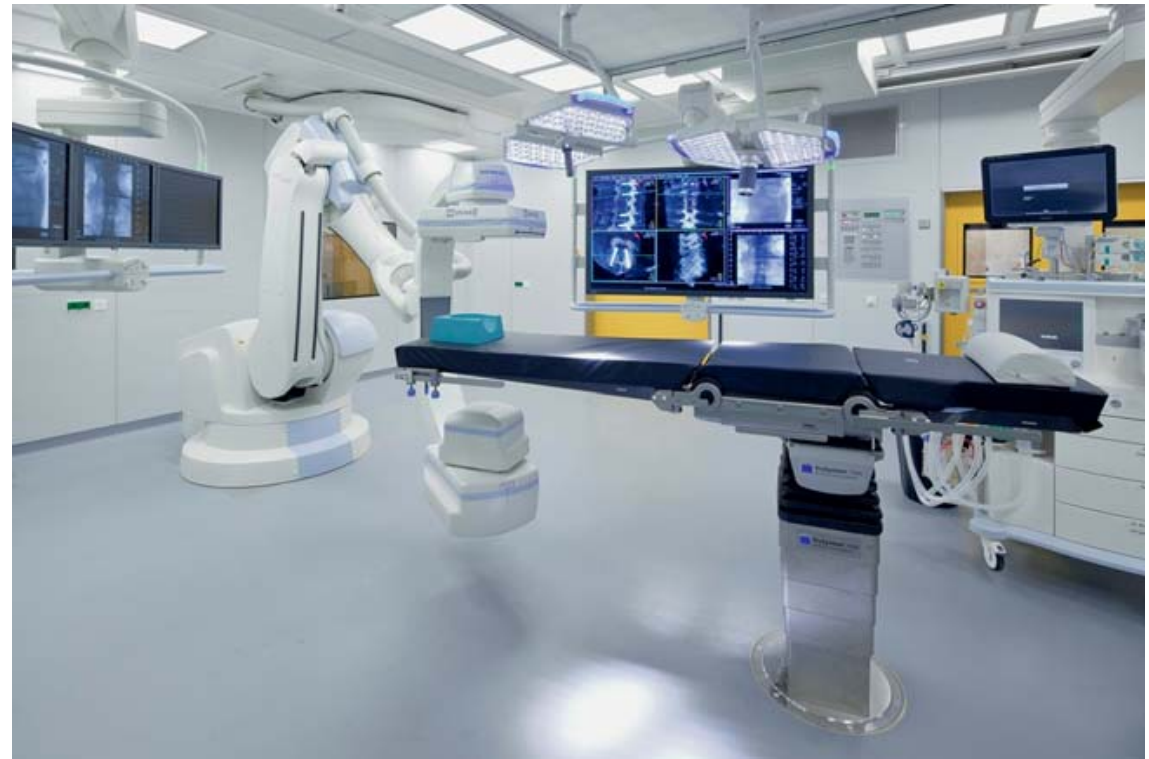

Abb. 13 Der Hybridoperationssaal (OP) verfügt über einen optimalen disziplinübergreifenden Aufbau. Zentrales Element dieses OPs ist das stationäre Bildgebungssystem mit dem integrierten Operationstisch.

teraler Kippung könnten als Kompromiss in Erwägung gezogen werden. Die höchste Nutzungsflexibilität kann jedoch nur durch den Einbau eines OP-Systemtischs erreicht werden. Eine detaillierte Analyse der Arbeitsabläufe im OP ist erforderlich, in die alle klinischen Disziplinen, die Anästhesiologie und das Pflegepersonal involviert sein müssen. Die optimale OP-Position des Patienten muss sowohl in Bezug auf die Narkose als auch auf das Bildgebungssystem ausgewertet werden. Sterilität muss ständig gewährleistet sein, solange der Chirurg, das Pflegepersonal und die Anästhesie noch Zugang zum Patienten brauchen (Abb. 14 und 15).

\section{Fixer C-Bogen mit Flat-Panel-Technologie}

Die C-Bogen-CT (Siemens syngo DynaCT) ist ein neues und innovatives Bildgebungsverfahren, bei dem durch ein CBogen-Angiografie-System mit Flachdetektor eine Reihe von 2-dimensionalen (2-D) Röntgenprojektionen gewonnen werden, um CT-ähnliche Bilder zu erzeugen [5].

Der C-Bogen rotiert innerhalb von ein paar Sekunden um den Patienten und akquiriert dabei hunderte von Projektionen, die den 3-D-Cone-Beam-Rekonstruktionen als Input dienen. Zur artefaktfreien Rekonstruktion benötigt man normalerweise mind. einen Scanwinkelbereich von $180^{\circ}$ zusätzlich zum Öffnungswinkel der Röntgenröhre. Dies bedeutet, dass für typische Angiografiesysteme ein Scan- winkelbereich von mind. $200^{\circ}$ Voraussetzung ist. Die anfallenden Volumendatensätze können entweder als Schnittbilder angezeigt werden oder als 3-D-Datensätze unter Verwendung der verschiedenen Volume Rendering Techniques (VRT) [2]. Die räumliche Auflösung, die syngo DynaCT bietet, kann im Sub-MillimeterBereich liegen. Zweckbestimmte Protokolle und $1 \times 1$-Binning machen Auflösungen von 0,1 mm möglich (Abb. 16).

Die Detektorgröße von $30 \times 40 \mathrm{~cm}$ $\left(12^{\prime \prime} \times 16^{\prime \prime}\right)$ macht die Erfassung eines großen Organs oder bspw. die Visualisierung des gesamten Beckens inkl. der beiden Beckenarterien mit nur einem Bild möglich (Abb. 17).

Die Möglichkeit der Bildzusammensetzung durch das Zusammenfügen von 2D-Durchleuchtungsbildern verhilft $\mathrm{zu}$ einer Gesamtdarstellung der Wirbelsäule.

CT-ähnliche Weichteilbildqualität kann im Hybrid-OP, abhängig vom Aufnahmeprotokoll, durch eine Kontrastauflösung von bis zu 3-5 HU erreicht werden (im Vergleich zu $1 \mathrm{HU}$ bei einer herkömmlichen $(T)$.

Die Möglichkeit der Bildfusion mit jeglichen präoperativen Bilddaten, also CT oder MR, erweitert den auf größtmögliche Präzision und Zielführung ausgelegten Operationsplan (Abb. 18). 


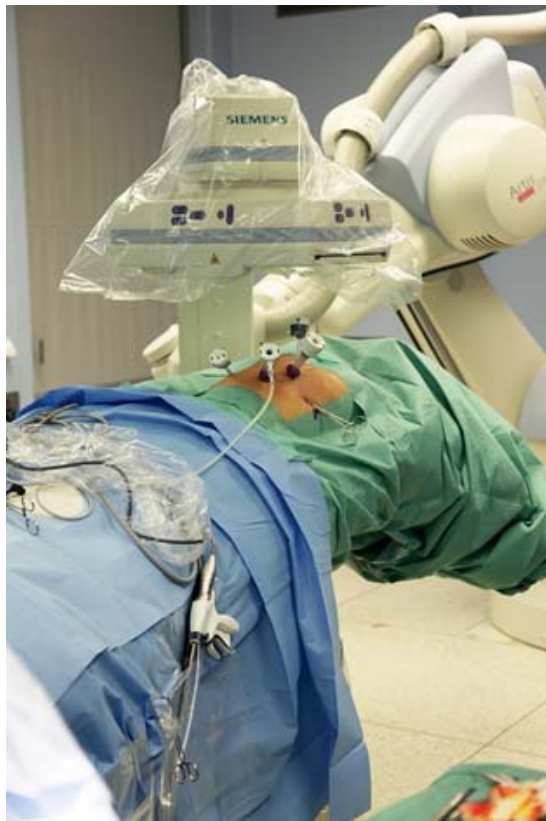

Abb. 14 Die Position des Patienten und die sterile Abdeckung sind ausschlaggebend für den Erfolg eines Hybrid-OPs. Für eine laparoskopische Leberteilresektion muss z.B. der Tisch hochgestellt (der Kopf zeigt zum Bildgebungssystem) und nach links gedreht werden, um den bestmöglichen Zugang zum Zielorgan zu garantieren.

\section{Intraoperative 3-D-Bildgebung}

Die Integration von 3-D-Bildgebungstechnik in routinemäßige klinische $\mathrm{Ar}$ beitsabläufe im OP bedarf eines gut durchdachten Aufbaus. Da sich die Position der Geräte und des Personals je nach Art des Eingriffs verändert, ist die Adaptionsfähigkeit des Bildgebungssystems von Bedeutung, damit es sich an die spezifischen Bedingungen im OP anpassen kann.

Die Integration des Bildgebungssystems in einen OP-Tisch ermöglicht die Bildgebung auch bei komplizierten chirurgischen Lagerungen, sodass keine Umlagerung für die Aufnahme nötig ist.

Während der Raum und die chirurgischen Instrumente besondere Anforderungen erfüllen müssen, um MRI-kompatibel zu sein, und den freien Zugang zum Patienten während einer CT im OP beeinträchtigen können, hat das Angiografiesystem besondere Vorteile in der Patientenbehandlung gezeigt und bietet die meiste Flexibilität. Neben der $\mathrm{Zu}-$ gänglichkeit und der Patientenlagerung hat sich die Bildqualität als ausreichend und präzise erwiesen [4].

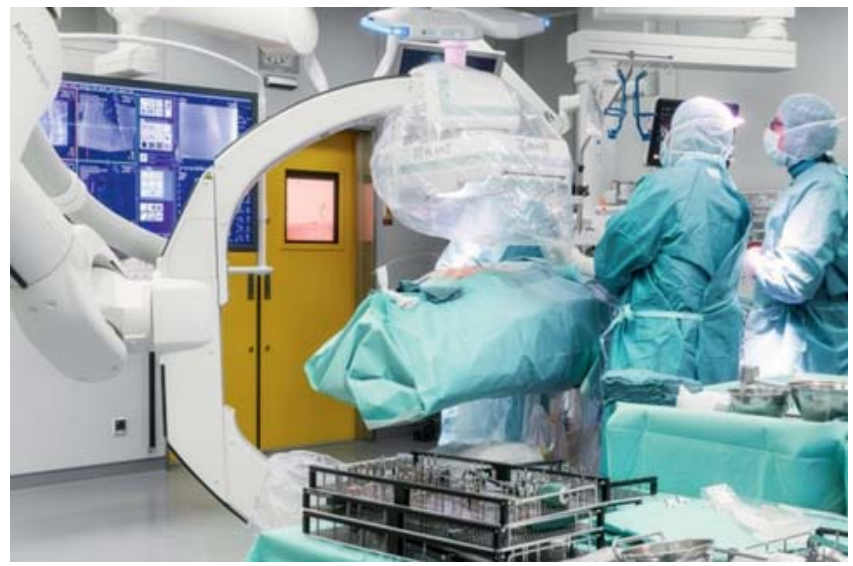

Abb. 15 Für einen minimalinvasiven Eingriff an der Wirbelsäule wird der Patient in Bauchlage gebracht, mit dem Kopf vom Narkosegerät abgewandt, welches sich am Fuß des Tisches befindet. Der Patient ist somit optimal positioniert.

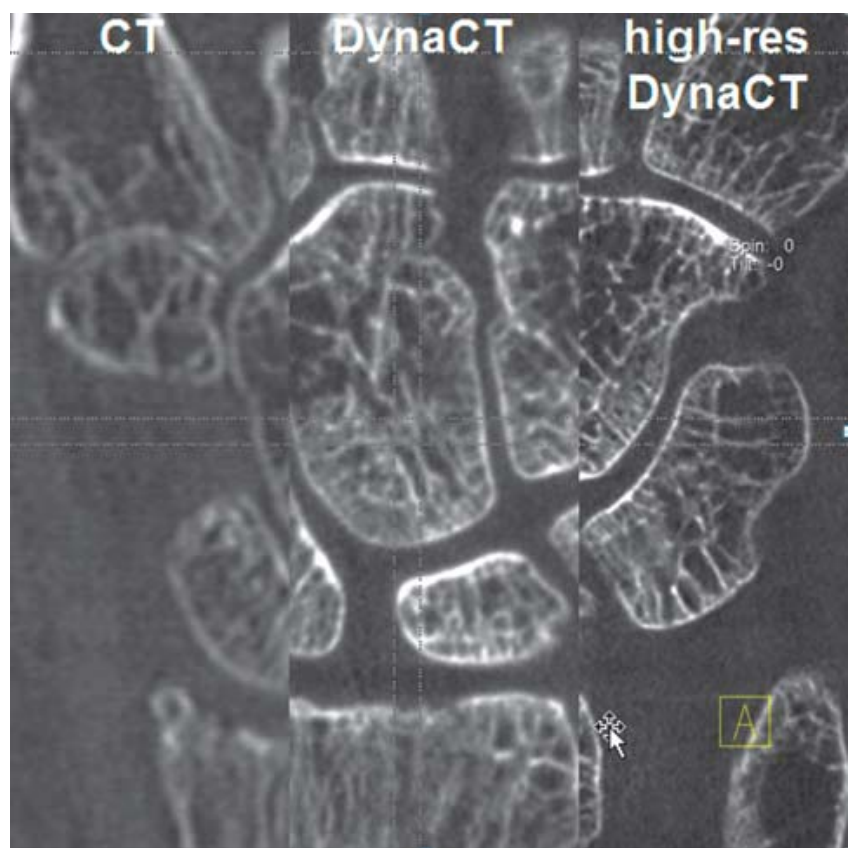

Abb. 16 Vergleich der Kontrastauflösung zwischen $\mathrm{CT}$ und Cone-BeamCT (syngo DynaCT). Das hochauflösende syngo DynaCT ermöglicht eine Auflösung von $0,1 \mathrm{~mm}$.

Die Sterilität des Operationsfelds muss während der 3-D-Aufnahmen gewährleistet bleiben. Die Abdeckung des Patienten mit einer zusätzlichen sterilen OP-Decke und das Befestigen der Decke rund um den Tisch wahrt die Sterilität und minimiert das Infektionsrisiko für den Patienten (Abb. 19).

Während der 3-D-Aufnahmen rotiert der C-Bogen, je nach Bildgebungsprotokoll und Apparat, 3-20 s lang um den Patienten. Der Bediener steuert die Aufnahme von einem steril abgedeckten Kontrollpult am Tisch oder von einem Gerätewagen.

\section{Visualisierung von 3-D-Röntgen- oder Angiografiebildern}

Eine Visualisierung in 3-D ergänzt 2-DProjektionsbilder um wertvolle Informa- tionen, wenn es darauf ankommt, komplexe Anatomie oder Pathologie, wie z.B. gebrochene Wirbel oder rupturierte zerebrale Aneurysmen, verständlich zu machen. Die Strukturen befinden sich auf einem Datenträger und können durch das Generieren von Querschnitten (doppelt schräg), durch Rotieren oder Ausschneiden beliebig betrachtet werden. Somit werden die räumlichen Beziehungen von Strukturen deutlich und die perspektivischen Verkürzungen, die bei 2-D-Aufnahmen sichtbar sind, können überwunden werden (Abb. 20). Während 2-D-Aufnahmen aus Pixeln innerhalb einer Ebene bestehen, besteht die 3-D-Datei aus Voxeln, d.h. kleinsten Einheiten als Träger der Bildinformation. 

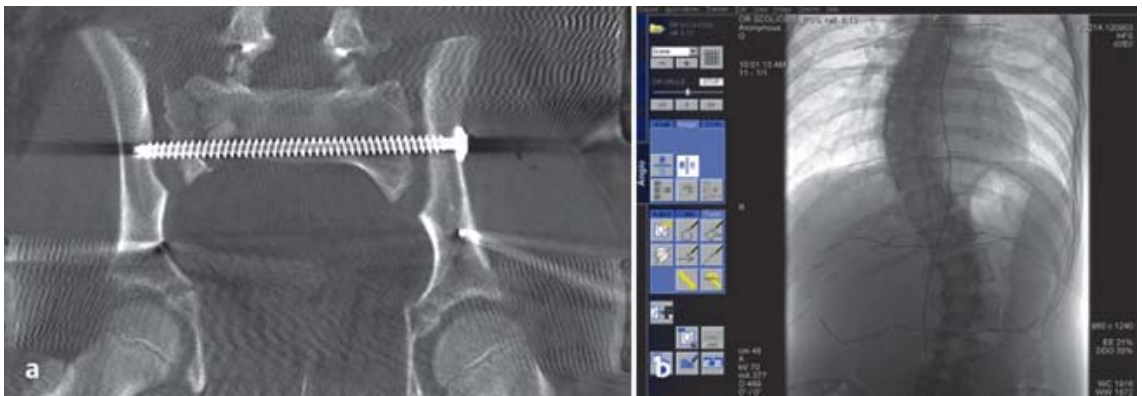

Abb. 17a und b a Der große Flachdetektor ermöglicht dem Chirurgen die Erfassung des gesamten Beckens mit einem einzigen Bild. b Stitching-Protokolle helfen dabei, die Gesamtdarstellung des Zielbereichs zu verbessern.
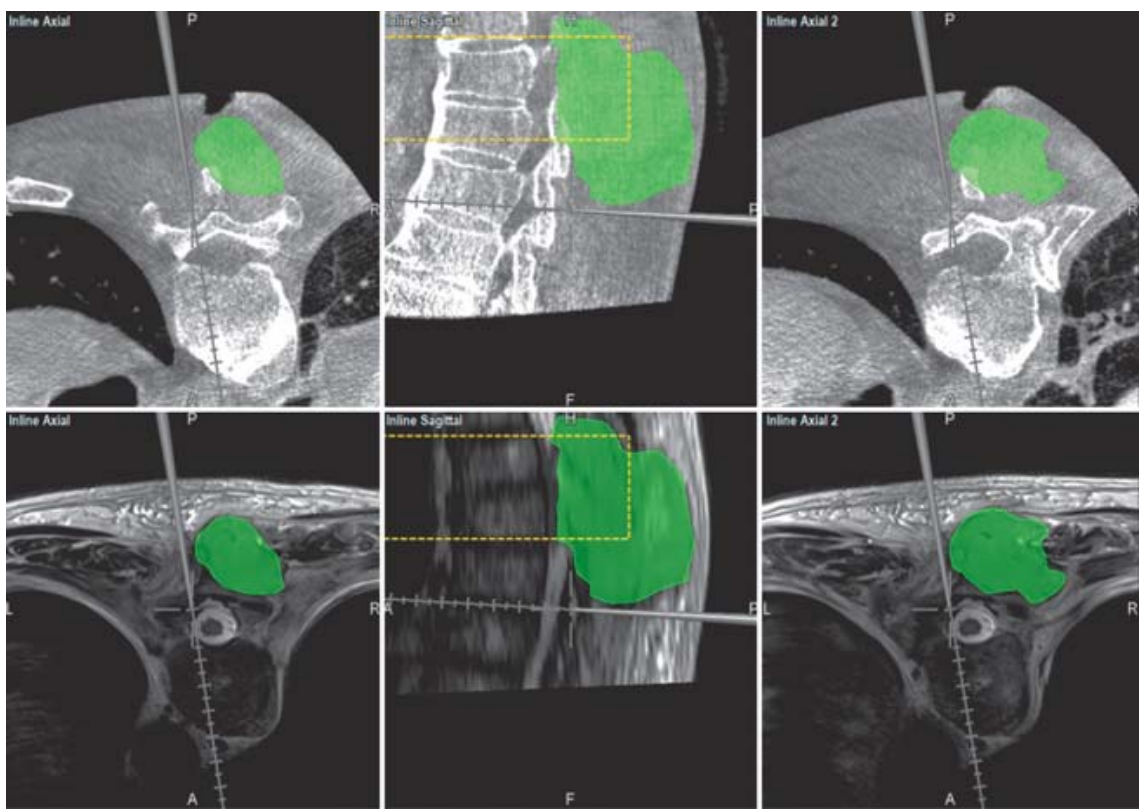

Abb. 18 Intraoperative MR- und syngo DynaCT-Fusion zur Durchführung einer navigationsgestützten muskuloskelettalen Tumoroperation. Die Bildfusion gibt dem Chirurgen mehr Information und erleichtert die Entscheidungsfindung.

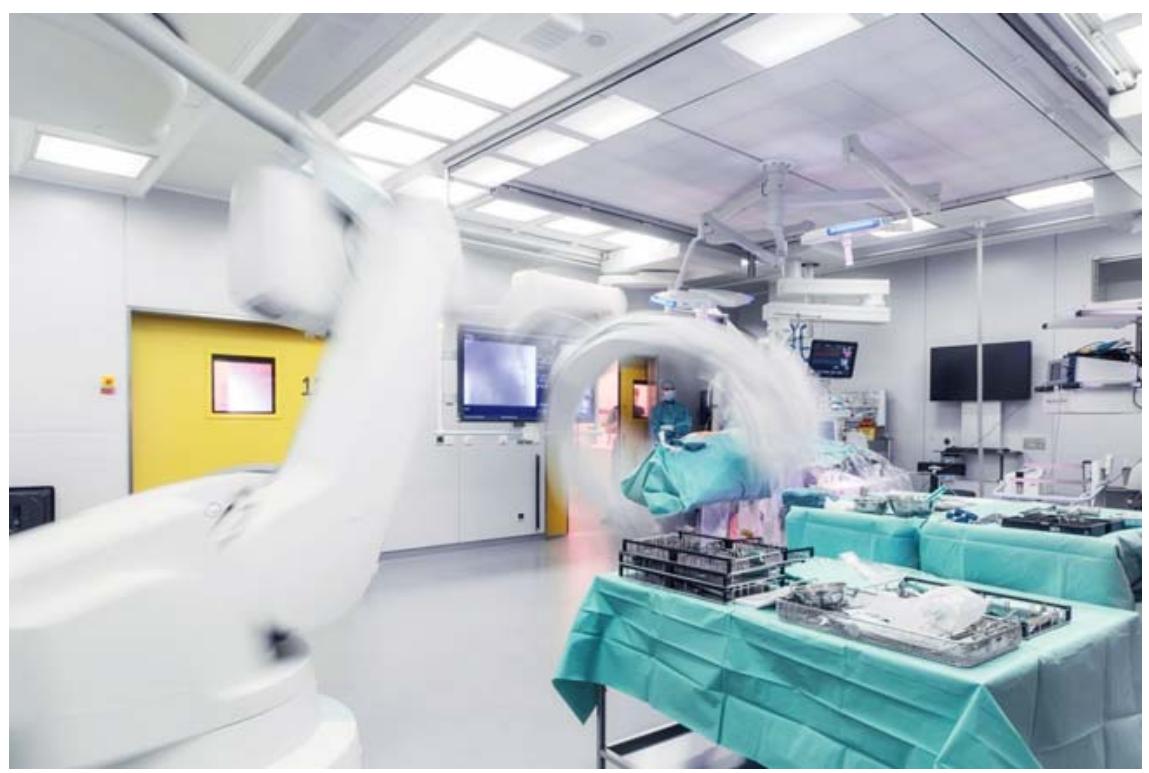

Abb. 19 Die Flexibilität des Systems, der Erhalt der Sterilität und die eigenständige Nutzung vom Chirurgen gewährleisten eine schnelle und präzise Behandlung.

\section{Navigation und Zielführung}

Intraoperative 3-D-Bildgebung kann auch in Verbindung mit Navigationssystemen, die die Nachverfolgung von Instrumenten oder externen Markern durchführen, genutzt werden. Die Navigationstechnologie hat sich bei chirurgischen Disziplinen mit starren Strukturen bewährt, so z. B. in der orthopädischen Chirurgie, der Mund-, Kiefer- und Gesichtschirurgie (MKG) und der Neurochirurgie [1].

Der Großteil der chirurgischen Navigationssysteme basiert auf optischer Trackingtechnologie. Daneben werden zunehmend Systeme entwickelt, die elektromagnetisches Tracking unterstützen. Elektromagnetisches Tracking eliminiert das Problem der Sichtverbindung. Außerdem kann der Lagesensor in die Spitze des Geräts integriert werden. Dies ermöglicht die Nutzung dieser Trackingtechnologie bei flexiblen Instrumenten. Diese beiden Vorteile werden die zukünftigen, auf dieser Technologie basierenden Entwicklungen vorantreiben.

Navigationssysteme müssen in die intraoperativen Bildgebungsverfahren integriert werden, um einen optimierten $\mathrm{Ar}$ beitsablauf zu schaffen.

Diese Integration beinhaltet die Bildübertragung und reibungslose Registrierungstechniken. Zwei wesentliche Faktoren beeinflussen die Genauigkeit der chirurgischen Navigation. Beim 1. Faktor handelt es sich um die Genauigkeit der Registrierung des 3-D-Datenträgers beim Navigationssystem. Intraoperative Bildgebungsverfahren bieten den Vorteil eines automatisierten Registrierungsprozesses, wodurch die Fehler menschlichen Handelns minimiert werden (Abb. 21). Die äußerst reproduzierbaren Bewegungsabläufe eines fixen C-BogenSystems (z.B. Artis zeego) unterstützen diesen Prozess durch die Integration des OP-Tisches. Somit kennt der C-Bogen seine Position in Relation zum Tisch und verfügt über einen größeren Bildausschnitt und einen hohen Dynamikbereich. Darüber hinaus können die Positionen des C-Bogens mit einem einzigen Klick gespeichert und wieder abgerufen werden.

Beim 2. wesentlichen Faktor, der für höchste Präzision entscheidend ist, handelt es sich um die Richtigkeit der für die Navigation verwendeten 3-D-Daten. Es gibt viele erdenkliche Gründe, weshalb 


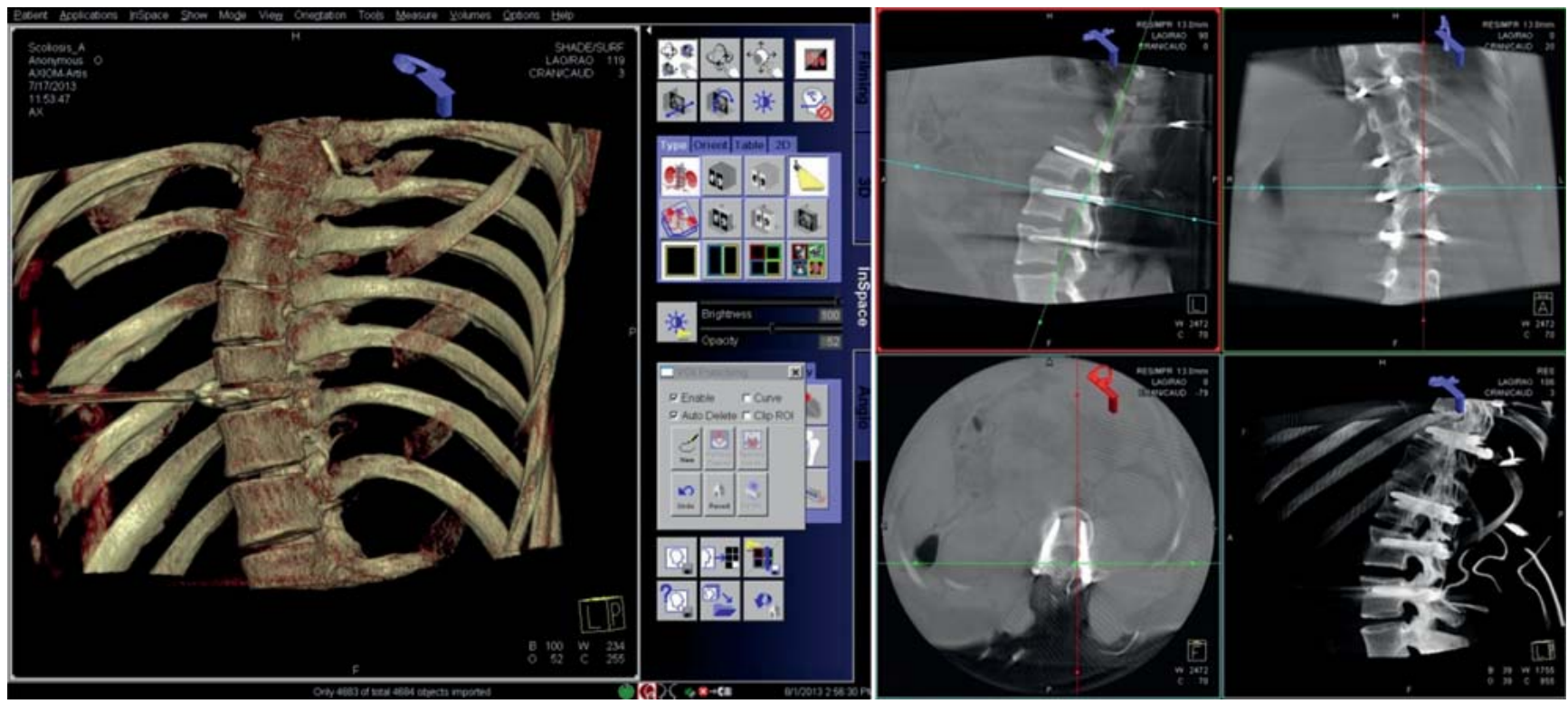

Abb. 20 Die Visualisierung in 3-D zusätzlich zur Visualisierung verschiedener Ebenen in 2-D hilft dem Chirurgen dabei, Schrauben an der exakten Stelle zu positionieren. Sollten sich die Schrauben nicht an der exakten Position befinden, kann ihre Position, falls nötig, direkt im OP korrigiert werden. Mit freundlicher Genehmigung von Prof. Dr. Ebara, Fujisawa, Japan und Prof. Dr. Banse, Brüssel, Belgien.

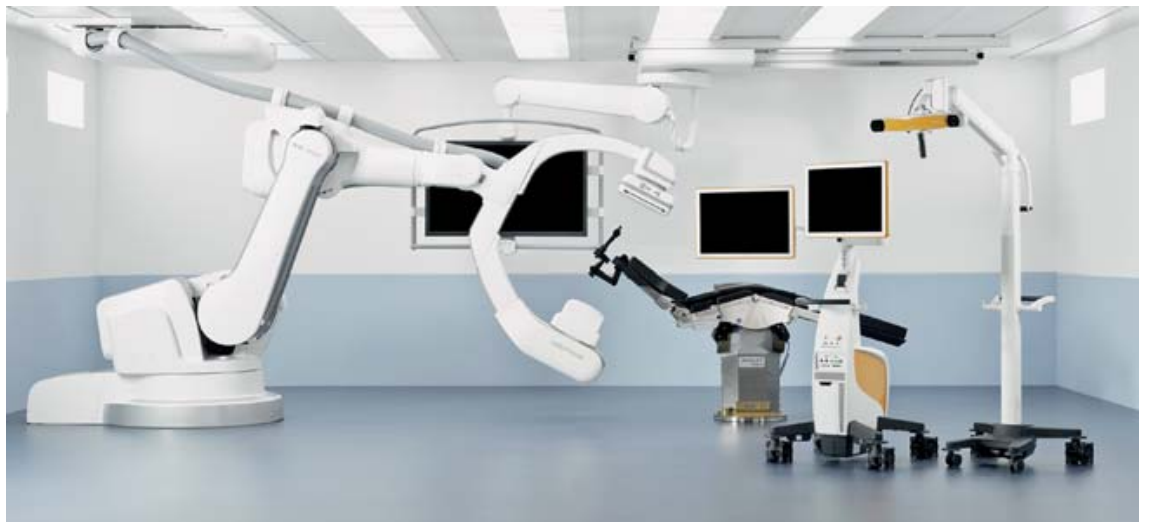

Abb. 21 Die Integration des Artis zeego in den Operationstisch und das Navigationssystem ermöglichen einen optimierten und beschleunigten Arbeitsablauf mit automatisierter Registrierung beim Navigationssystem.

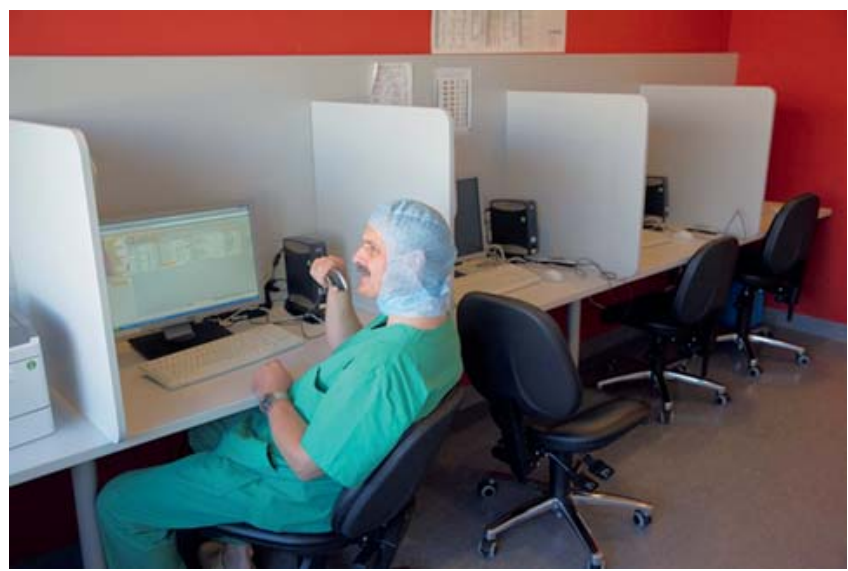

die Morphologie der bildgebenden Daten verändert sein kann, wenn diese nicht intraoperativ erworben wurden. $\mathrm{Zu}$ solchen Beispielen gehören Veränderungen der Wirbelsäulenmorphologie durch die Patientenlagerung oder BrainShift während einer Kraniotomie. Intraoperative 3-D-Bildgebung wird helfen, diese Herausforderungen zu meistern, indem sie die Bilder für Navigationszwecke durch aktuelle, hochwertige 3D-Scans auf den neuesten Stand bringt. Diese Updates können auch gemacht werden, indem man eine auf einem intraoperativen Scan basierende, nicht rigide Deformation auf die präoperativen Bilder anwendet.

Abb. 22 Effizientere Bildgebungsverfahren bedeuten mehr Zeit für den Chirurgen.

\section{Schlussfolgerung}

Die Flachdetektortechnologie bietet präzisere Bilder, ein größeres Sichtfeld, weniger Verzerrungen und mehr Platz für chirurgische Eingriffe und Instrumente.

Zudem werden die Flachdetektor-C-Bögen zunehmend erschwinglich und haben gegenüber den Bildverstärkern die Vorteile der Motorisierung und höheren Präzision. Innovationen bei mobilen CBögen mit Flachdetektortechnologie sind aktive Kühlsysteme für längere Eingriffe, Motorisierung, Bedienfelder für den Chirurgen sowie eine Betriebsart mit hoher Leistung für adipöse Patienten. 
Gerade für komplexe Verletzungen, schwierige anatomischen Regionen oder anspruchsvolle minimalinvasive Operationsverfahren ist der Hybridoperationssaal geeignet. Durch einen intraoperativen Scan ist bspw. das komplette Becken visualisierbar. Des Weiteren zeichnet sich der Hybrid-OP durch eine maximale Selbstständigkeit des Chirurgen aus, da der C-Bogen vollständig durch den Operateur direkt am Operationstisch bedient werden kann.

Gerade im Rahmen der Polytraumaversorgung ist der Hybrid-OP aufgrund seiner multidisziplinären Anwendbarkeit hervorragend geeignet. Mit all diesen Funktionen kann sich der chirurgische Workflow mit modernen C-Bögen mit Flachdetektortechnologie signifikant verbessern (Abb. 22). Die Zukunft der intraoperativen Bildgebung liegt in der Flachdetektorbildgebung.

\section{Literatur}

${ }^{1}$ Gelalis ID, Paschos NK, Pakos EE et al. Accuracy of pedicle screw placement: a systematic review of prospective in vivo studies comparing free hand, fluoroscopy guidance and navigations techniques. Eur Spine J 2012; 21 : 247-255
${ }^{2}$ Kalender W, Kyriakou Y. Flat-detector computed tomography (FD-CT). Eur Radiol 2007; 17: 2767-2779

${ }^{3}$ Naim C et al. Special Topics in Cardiac Surgery 2012. Im Internet: http://www.intechopen. com/books/special-topics-in-cardiac-surgery/ the-hybrid-operating-room

${ }^{4}$ Nozaki T, Iida H, Morii A et al. Efficacy of laparoendoscopic single-site biopsy for diagnosis of retroperitoneal tumor of unknown origin. Urol Int 2013; 90: 95-100

5 Tomaszewski R. Planning a better operating room suite: design and implementation strategies for success. Periop Nursing Clin 2008; 3: 43-54

\section{Dr. med. Peter Hinnerk Richter} Assistenzarzt

Klinik für Unfall-, Hand-, Plastische und Wiederherstellungschirurgie Universität Ulm

Albert-Einstein-Allee 23

89081 Ulm

\section{Dr. med. Alexander Grafenberg} Clinical Education and Collaborations Manager

Healthcare

Siemens AG

Hartmannstraße 16

91052 Erlangen
Michael Bodky

Marketing Manager Surgery and Urology

Healthcare

Siemens AG

Hartmannstraße 16

91052 Erlangen

\section{Dr. Christian Schmidgunst}

Research and Development X-Ray Detectors

Healthcare

Siemens AG

Allee am Röthelheimpark 2

91052 Erlangen

\section{Dr. med. Ina Schwabenland}

Global Marketing Manager Hybrid OR's

Healthcare

Siemens AG

Siemensstraße 1

91301 Forchheim 